\title{
AIDS e grau de escolaridade no Brasil: evolução temporal de 1986 a 1996
}

\author{
AIDS and level of education in Brazil: \\ temporal evolution from 1986 to 1996
}

\author{
Maria Goretti Fonseca 1 \\ Francisco Inácio Bastos 2 \\ Monica Derrico 2 \\ Carla L. Tavares de Andrade 2 \\ Cláudia Travassos 2 \\ Celia Landmann Szwarcwald 1
}

\footnotetext{
1 Coordenação Nacional de DST e AIDS,

Ministério da Saúde. Esplanada dos Ministérios, Bloco G. Edifício sede, sobrel oja. Brasília, DF 70058-900, Brasil.

2 Departamento de Informações em Saúde, Centro de Informação Científica e Tecnológica, Fundação Oswaldo Cruz. Av. Brasil 4365, Rio de Janeiro, RJ 21045-900, Brasil.
}

\begin{abstract}
This articleanalyzes the temporal distribution of reported AIDS cases by level of education used as a proxy variable for individual socioeconomic status. All AIDS cases aged 20-69 years and reported through May 29, 1999, with date of diagnosis between 1986-1996, were included in the study. Incidence rates were calculated for men and women according to level of education ("level 1" up to 8 years of schooling and "I evel 2" with over 8 years of schooling), by five geographic regions, and by year of diagnosis. Incidence rates for men with less schooling were close to or higher than those for men with more schooling (particularly in the Southeast region). For women, a time series showed that incidence rates increased at a higher rate among women with less schooling in all regions of the country; in the Southeast, the incidence rate for women with less schooling was al ready greater than for women with more schooling by 1989. According to the present analysis, the AIDS epidemic in Brazil began among people from the more highly educated social strata and progressed steadily through to the less educated social strata, especially among women.
\end{abstract}

Key words Acquired Immunodeficiency Syndrome; Educational Status; Social status

Resumo Este trabalho analisa a evolução temporal dos casos de AIDS por grau de escolaridade como variável identificadora do nível sócio-econômi co do caso. Todos os casos de AIDS em indivíduos com idades entre 20 e 69 anos, notificados até 29 de maio de 1999, diagnosticados entre 1986 e 1996, foram incluídos no estudo. As taxas de inci dência para ambos os sexos foram calculadas segundo dois graus de escol ari dade - "grau 1" (casos com até oi to anos de estudo) e "grau 2" (com mais de oito anos de estudo) -, por regi ão e ano de diagnóstico. Entre os homens, as taxas de inci dência de AIDS para aqueles com menor escolaridade ul trapassam (sendo mais evidente na Regi ão Sudeste) ou se aproxi mam das taxas dos homens com maior escol aridade. Para as mulheres, a evolução temporal das taxas mostrou mai or ritmo de cresci mento entre as mulheres de menor escolaridade para todas as regiões, sendo que, na Sudeste, as taxas entre as mulheres com menor escolaridade ultrapassa as taxas daquel as com maior escolaridade em anos anteriores a 1989. Concluindo, a presente anál ise mostra, de forma consistente, que a epidemia de AIDS no Brasil se ini ci ou nos estratos sociais de mai or escolaridade, com progressiva disseminação para os estratos sociais de menor escolaridade.

Palavras-chave Síndrome da Imunodeficiência Adquirida; Escolaridade; Classe Social 
Introdução

Vários estudos têm apontado que a posição do indivíduo na estrutura social constitui um importante preditor das suas condições de saúde, sendo que o padrão de risco observado é constantemente desvantajoso para os indivíduos pertencentes aos grupos sociais menos privilegiados (Eames et al., 1993; Mustard et al., 1997; Sloggett \& Joshi, 1998).

Dentre os indicadores mais importantes para mensurar o nível sócio-econômico associado à saúde da população, citam-se o nível de instrução, a renda e a ocupação (Kunst \& Mackenbach, 1994a; Sorlie, 1995; Cairney \& Arnold, 1998). Kunst \& Mackenbach (1994b) sugerem que essas variáveis têm em comum o fato de evidenciarem a estratificação social: os indivíduos ocupam uma posição na hierarquia social de acordo com a sua ocupação, renda e nível educacional, sendo que cada um desses indicadores cobre um aspecto diferente da estratificação social. O nível educacional expressa diferenças entre pessoas em termos de acesso à informação e perspectivas e possibilidades de se beneficiar de novos conhecimentos; a renda representa antes de tudo o acesso aos bens materiais, inclusive aos serviços de saúde; e o status ocupacional inclui esses dois aspectos, além dos benefícios adquiridos em algumas profissões, tais como prestígio, privilégios e poder.

Os autores estabelecem ainda que, embora seja preferível examinar todos os indicadores simultaneamente em vez de apenas um, de maneira isolada, o nível educacional é considerado como o melhor, por ser este um indicador fácil de ser obtido e ter uma grande importância como determinante de saúde. Outra questão relevante é que a qualificação educacional é praticamente inalterável por todo o curso da vida adulta (Arber, 1996) e que, por isso, exceção feita ao uso desse indicador em pediatria (quando doenças importantes podem determinar dificuldades escolares, em um processo em curso), trata-se de um indicador praticamente impermeável às perdas que as doenças impõem em termos de renda ou status, questão particularmente relevante em se tratando da AIDS, associada comumente ao preconceito e ao estigma.

A escolaridade tem sido apontada como importante determinante da situação de saúde do indivíduo por vários autores (Luepker et al., 1993; Pappas et al., 1993; Mackenbach et al., 1997; Gulliford \& Mahabir, 1998), sendo inclusive a escolaridade da mãe importante preditor da mortalidade de seus filhos (Macharelli \& Oliveira, 1991).
No Brasil, a influência da escolaridade sobre as condições de saúde foi evidenciada pelos resultados da Pesquisa sobre Padrões de Vida (PPV), realizada em 1996/ 1997 pela Fundação Instituto Brasileiro de Geografia e Estatística (IBGE). Analisando-se a importância das variáveis sócio-econômicas na percepção do estado de saúde de indivíduos com vinte anos ou mais, como anos de estudo, ocupação e renda mensal, observou-se que a variável "anos de estudo" foi a segunda mais relevante, após a idade (Fonseca et al., 1999).

No tocante à infecção pelo HIV/ AIDS, a idéia de analisar a doença por uma perspectiva social, além das tradicionais categorias de exposição definidas a partir de comportamentos individuais, ganha impulso fundamental a partir do trabalho de Mann et al. (1992). Diversos outros autores (ver artigos de Bastos \& Szwarcwald e de Parker \& Camargo J r., neste fascículo) vêm analisando de forma sistemática as correlações entre a difusão do HIV/ AIDS e a pauperização das comunidades, a ruptura dos laços de interação social e a disponibilidade de recursos para as comunidades do ponto de vista da saúde, habitação e políticas públicas (Wallace, 1988, 1990).

Tendo em vista que a epidemia de AIDS no Brasil, após inicialmente atingir as metrópoles, vem apresentando um padrão de crescimento em direção a municípios cada vez de menor tamanho populacional (e de menor renda per capita), juntamente com o aumento do papel relativo da transmissão heterossexual (Szwarcwald et al., 1997), conjecturas sobre a pauperização da epidemia brasileira têm sido sugeridas.

Investiga-se, neste trabalho, a evolução temporal dos casos notificados de AIDS segundo o grau de escolaridade, variável identificadora do nível sócio-econômico do caso.

\section{Metodologia}

Todos os casos de AIDS em pessoas com idade entre 20 e 69 anos notificados à Coordenação Nacional de DST e AIDS do Ministério da Saúde até 29 de maio de 1999 e com data de diagnóstico entre 1986 e 1996 foram incluídos no estudo. O ano de 1996 foi o último ano considerado para análise, de modo a reduzir o efeito do atraso de notificação (Barbosa \& Struchiner, 1997).

O presente trabalho analisa os dados por categoria de escolaridade. Os casos notificados são rotineiramente classificados quanto à escolaridade em: analfabeto, ensino fundamental, ensino médio, ensino superior (universitário) e escolaridade ignorada. Para fins desta 
análise, estas categorias originais foram reagrupadas em três categorias de escolaridade: "1o grau", constituída pelos indivíduos com graus de escolaridade analfabeto e ensino fundamental; "2o grau”, para ensino médio e ensino superior; "ignorado", para escolaridade ignorada.

Foram feitas análises, para cada sexo e região, da evolução temporal das proporções em cada categoria de escolaridade. Devido à evolução irregular no tempo da proporção de casos com escolaridade ignorada em cada região, além da sua grande magnitude, foi necessário tratamento específico de tais casos.

Os casos com escolaridade ignorada foram tratados de duas formas. Na primeira, foram distribuídos proporcionalmente tal como os casos com escolaridade informada. Na segun$\mathrm{da}$, os casos com escolaridade ignorada foram reclassificados através de um modelo logístico de análise discriminante (Hosmer \& Lemeshow, 1989). Tal procedimento foi realizado por sexo e região, levando em conta a idade, categoria de exposição, ano de diagnóstico, ocupação, tempo de sobrevida após diagnóstico e presença das seguintes doenças e/ ou sinais no momento da notificação: tuberculose (qualquer forma), sarcoma de Kaposi, pneumonia por Pneumocystis carinii e caquexia, com objetivo de discriminar os dois grupos com escolaridade informada. A metodologia pormenorizada deste procedimento será objeto de publicação específica.

A evolução temporal das proporções dos casos por escolaridade nas duas categorias " 1 으 grau" e "2o grau", após a correção dos casos com escolaridade ignorada por ambos os procedimentos, foi avaliada através do cálculo das taxas de variação anual das proporções dessas categorias de escolaridade, ajustadas por regressão exponencial, com o tempo, nos períodos de, respectivamente, 1989 a 1996, para o sexo masculino, e de 1990 a 1996, para o sexo feminino. Testes estatísticos foram aplicados aos coeficientes angulares para verificar significâncias das tendências temporais.

Para a Região Norte, foi considerado o período de 1991 a 1996, para ambos os sexos, em virtude das oscilações do número de casos nos primeiros anos da epidemia nessa região, o que poderia afetar as tendências estabelecidas.

Para apreciação do comportamento temporal dos riscos por escolaridade foram calculadas taxas de incidência segundo grau de escolaridade e sexo, em cada região, por ano de diagnóstico. Os períodos de tempo considerados foram os mesmos descritos para a análise das proporções.
Os numeradores das taxas foram constituídos respectivamente pelos casos agrupados em "1o grau" e "2o grau", acrescidos dos casos originalmente com escolaridade ignorada e reclassificados pelo modelo de análise discriminante.

Os denominadores das taxas de incidência foram estimados através dos dados do Instituto Brasileiro de Geografia e Estatística. Primeiramente, por meio das informações sobre população no Censo Demográfico de 1991 e na Contagem da População de 1996, foram estimadas as populações de 20 a 69 anos de idade por sexo e região no período de 1987 a 1996, por meio de interpolação geométrica.

Para estimar as populações de acordo com a categorização da escolaridade utilizada para os casos de AIDS sob análise, Iançou-se mão das proporções da população pelo número de anos de estudos que constavam do Censo Demográfico de 1991 correspondentes às duas categorias previamente definidas. Essas proporções foram, então, aplicadas às populações projetadas, no período de 1987 a 1996, para a obtenção dos denominadores.

Para o seguimento temporal da evolução das taxas de incidência por categoria de escolaridade nos períodos 1989-1996 (sexo masculino) e 1990-1996 (sexo feminino), procedeu-se de forma análoga à metodologia descrita para a avaliação do comportamento das proporções no tempo.

\section{Resultados}

Foram analisados 115.439 casos de AIDS em pessoas com idade entre 20 a 69 anos, diagnosticados entre 1986 e 1996, sendo $91.978(79,7 \%)$ do sexo masculino.

As Tabelas 1a, 1b e 2a, 2b apresentam os casos notificados de AIDS para os sexos masculino e feminino, segundo região de residência e categoria de escolaridade, por ano de diagnóstico. Nas Tabelas la e 1b, os casos com escolaridade ignorada foram proporcionalmente distribuídos entre as duas categorias com escolaridade informada, enquanto nas Tabelas $2 a$ e $2 \mathrm{~b}$, os casos com escolaridade ignorada foram corrigidos através de um modelo de análise discriminante.

Para o sexo masculino, percebe-se uma tendência nitidamente crescente para as proporções de casos classificados em escolaridade “10 grau", atingindo, para o Brasil, níveis próximos a 70\%, no último ano em análise. As diferenças inter-regionais não se mostraram tão marcantes como as encontradas por sexo. Para as mu- 
Distribuição (\%) dos casos notificados de AIDS em homens de 20 a 69 anos de idade por grau de escolaridade e ano de diagnóstico e taxa de variação anual segundo as grandes regiões. Brasil, 1989-1996.

\begin{tabular}{|c|c|c|c|c|c|c|c|c|c|c|}
\hline \multirow[t]{2}{*}{ Escolaridade } & \multicolumn{8}{|c|}{ Ano de diagnóstico } & \multirow{2}{*}{$\begin{array}{l}\text { Taxa de variação } \\
\text { anual (\%) }\end{array}$} & \multirow[t]{2}{*}{$\mathrm{p}$} \\
\hline & 89 & 90 & 91 & 92 & 93 & 94 & 95 & 96 & & \\
\hline \multicolumn{11}{|l|}{ Região Norte } \\
\hline 1으 Grau & - & - & 43,9 & 48,6 & 50,0 & 51,7 & 59,4 & 67,6 & 8,31 & 0,002 \\
\hline $20 \mathrm{Grau}$ & - & - & 56,1 & 51,4 & 50,0 & 48,3 & 40,6 & 32,4 & $-9,47$ & 0,006 \\
\hline \multicolumn{11}{|c|}{ Região Nordeste } \\
\hline 1o $\mathrm{Grau}$ & 36,9 & 39,9 & 43,7 & 47,1 & 55,2 & 58,6 & 59,2 & 61,4 & 8,14 & 0,000 \\
\hline $2 \underline{o} \mathrm{Grau}$ & 63,1 & 60,1 & 56,3 & 52,9 & 44,8 & 41,4 & 40,8 & 38,6 & $-7,39$ & 0,000 \\
\hline \multicolumn{11}{|c|}{ Região Sudeste } \\
\hline 1으 Grau & 56,1 & 57,1 & 60,3 & 63,3 & 63,8 & 65,2 & 66,6 & 69,4 & 3,03 & 0,000 \\
\hline 2ㅇ Grau & 43,9 & 42,9 & 39,7 & 36,7 & 36,2 & 34,8 & 33,4 & 30,6 & $-4,87$ & 0,000 \\
\hline \multicolumn{11}{|l|}{ Região Sul } \\
\hline 10 Grau & 39,2 & 47,1 & 52,9 & 56,0 & 60,7 & 58,7 & 62,5 & 65,1 & 8,54 & 0,001 \\
\hline $2 \varrho \mathrm{Grau}$ & 60,8 & 52,9 & 47,1 & 44,0 & 39,3 & 41,3 & 37,5 & 34,9 & $-8,48$ & 0,000 \\
\hline \multicolumn{11}{|c|}{ Região Centro-O este } \\
\hline 1o Grau & 38,7 & 40,5 & 45,7 & 49,7 & 58,9 & 67,4 & 65,6 & 63,4 & 8,96 & 0,001 \\
\hline 2ㅇ Grau & 61,2 & 59,5 & 54,3 & 50,3 & 41,1 & 32,6 & 34,4 & 36,6 & $-8,28$ & 0,001 \\
\hline \multicolumn{11}{|l|}{ Brasil** } \\
\hline 10 Grau & 52,8 & 54,1 & 57,2 & 60,4 & 62,3 & 63,5 & 65,1 & 67,6 & 3,64 & 0,000 \\
\hline 2ㅇ Grau & 47,2 & 45,9 & 42,8 & 39,6 & 37,7 & 36,5 & 34,9 & 32,4 & $-5,26$ & 0,000 \\
\hline
\end{tabular}

* Os casos com escolaridade ignorada foram proporcionalmente distribuídos entre aqueles com escolaridade informada.

** Incluídos casos da Região Norte de 1989 e 1990.

Tabela $1 b$

Distribuição (\%) dos casos notificados de AIDS em mulheres de 20 a 69 anos de idade por grau de escolaridade e ano de diagnóstico e taxa de variação anual (\%) segundo as grandes regiões. Brasil, 1990-1996.

\begin{tabular}{|c|c|c|c|c|c|c|c|c|c|}
\hline \multirow[t]{2}{*}{ Escolaridade } & \multicolumn{7}{|c|}{ Ano de diagnóstico } & \multirow[t]{2}{*}{ Taxa de variação anual (\%) } & \multirow[t]{2}{*}{ p } \\
\hline & 90 & 91 & 92 & 93 & 94 & 95 & 96 & & \\
\hline \multicolumn{10}{|l|}{ Região Norte } \\
\hline 10 Grau & - & 53,8 & 72,0 & 78,4 & 73,3 & 74,6 & 78,2 & 5,60 & 0,104 \\
\hline 2o Grau & - & 46,2 & 28,0 & 21,6 & 26,7 & 25,4 & 21,8 & $-10,38$ & 0,095 \\
\hline \multicolumn{10}{|c|}{ Região Nordeste } \\
\hline 1o G rau & 67,2 & 67,7 & 62,5 & 68,6 & 72,3 & 70,4 & 73,7 & 1,79 & 0,076 \\
\hline 2o Grau & 32,8 & 32,3 & 37,5 & 31,4 & 27,7 & 29,6 & 26,3 & $-3,95$ & 0,055 \\
\hline \multicolumn{10}{|c|}{ Região Sudeste } \\
\hline 1o Grau & 76,8 & 79,9 & 77,5 & 79,4 & 79,0 & 78,7 & 79,0 & 0,25 & 0,376 \\
\hline $20 \mathrm{Grau}$ & 23,2 & 20,1 & 22,5 & 20,6 & 21,0 & 21,3 & 21,0 & $-0,86$ & 0,406 \\
\hline \multicolumn{10}{|l|}{ Região Sul } \\
\hline $10 \mathrm{Grau}$ & 62,3 & 67,7 & 67,5 & 69,1 & 66,7 & 67,7 & 73,6 & 3,33 & 0,003 \\
\hline 2o Grau & 37,7 & 32,3 & 32,5 & 30,9 & 33,3 & 32,3 & 26,4 & $-6,11$ & 0,007 \\
\hline \multicolumn{10}{|c|}{ Região Centro-O este } \\
\hline 10 G rau & 50,0 & 70,8 & 69,4 & 73,6 & 72,8 & 76,8 & 77,6 & 4,44 & 0,069 \\
\hline $20 \mathrm{Grau}$ & 50,0 & 29,2 & 30,6 & 26,4 & 27,2 & 23,2 & 22,4 & $-7,68$ & 0,061 \\
\hline \multicolumn{10}{|l|}{ Brasil** } \\
\hline 1ㅇ Grau & 73,6 & 76,9 & 75,0 & 77,0 & 76,3 & 76,2 & 77,4 & 0,54 & 0,108 \\
\hline $20 \mathrm{Grau}$ & 26,4 & 23,1 & 25,0 & 23,0 & 23,7 & 23,8 & 22,6 & $-1,63$ & 0,111 \\
\hline
\end{tabular}

* Os casos com escolaridade ignorada foram proporcionalmente distribuídos entre aqueles com escolaridade informada.

** Incluídos casos da Região Norte de 1990. 
Distribuição (\%) dos casos notificados de AIDS em homens de 20 a 69 anos de idade por grau de escolaridade e ano de diagnóstico e taxa de variação anual (\%) segundo as grandes regiões. Brasil, 1989-1996.

\begin{tabular}{|c|c|c|c|c|c|c|c|c|c|c|}
\hline \multirow[t]{2}{*}{ Escolaridade } & \multicolumn{8}{|c|}{ Ano de diagnóstico } & \multirow{2}{*}{$\begin{array}{l}\text { Taxa de variação } \\
\text { anual (\%) }\end{array}$} & \multirow[t]{2}{*}{$\mathrm{p}$} \\
\hline & 89 & 90 & 91 & 92 & 93 & 94 & 95 & 96 & & \\
\hline \multicolumn{11}{|l|}{ Região Norte } \\
\hline $10 \mathrm{Grau}$ & - & - & 45,0 & 54,5 & 57,1 & 57,9 & 63,4 & 71,1 & 8,15 & 0,003 \\
\hline 2o Grau & - & - & 55,0 & 45,5 & 42,9 & 42,1 & 36,6 & 28,9 & $-10,47$ & 0,003 \\
\hline \multicolumn{11}{|c|}{ Região Nordeste } \\
\hline 1o Grau & 37,4 & 42,6 & 46,2 & 51,4 & 61,4 & 63,0 & 65,6 & 68,0 & 9,28 & 0,000 \\
\hline 2o Grau & 62,6 & 57,4 & 53,8 & 48,6 & 38,6 & 37,0 & 34,4 & 32,0 & $-9,74$ & 0,000 \\
\hline \multicolumn{11}{|c|}{ Região Sudeste } \\
\hline 1으 Grau & 59,1 & 63,0 & 66,2 & 67,8 & 69,2 & 71,1 & 72,3 & 75,0 & 3,13 & 0,000 \\
\hline 2o Grau & 40,9 & 37,0 & 33,8 & 32,2 & 30,8 & 28,9 & 27,7 & 25,0 & $-6,22$ & 0,000 \\
\hline \multicolumn{11}{|l|}{ Região Sul } \\
\hline 10 Grau & 36,9 & 49,1 & 53,3 & 59,6 & 64,0 & 63,9 & 68,0 & 69,9 & 8,32 & 0,001 \\
\hline 2o Grau & 63,1 & 50,9 & 46,7 & 40,4 & 36,0 & 36,1 & 32,0 & 30,1 & $-9,50$ & 0,000 \\
\hline \multicolumn{11}{|c|}{ Região Centro-Oeste } \\
\hline $10 \mathrm{Grau}$ & 42,9 & 44,1 & 54,1 & 59,4 & 69,7 & 77,0 & 74,5 & 74,8 & 9,65 & 0,000 \\
\hline 2ㅇ G rau & 57,1 & 55,9 & 45,9 & 40,6 & 30,3 & 23,0 & 25,5 & 25,2 & $-13,32$ & 0,003 \\
\hline \multicolumn{11}{|l|}{ Brasil** } \\
\hline $10 \mathrm{Grau}$ & 54,8 & 59,0 & 62,3 & 65,0 & 67,8 & 69,5 & 71,1 & 73,4 & 4,08 & 0,000 \\
\hline 2 Grau & 45,2 & 41,0 & 37,7 & 35,0 & 32,2 & 30,5 & 28,9 & 26,6 & $-7,11$ & 0,000 \\
\hline
\end{tabular}

* Os casos com escolaridade ignorada foram corrigidos por um modelo de análise discriminante.

** Incluídos casos da Região Norte de 1990.

Tabela $2 b$

Distribuição (\%) dos casos notificados de AIDS em mulheres de 20 a 69 anos de idade por grau de escolaridade e ano de diagnóstico e taxa de variação anual (\%) segundo as grandes regiões. Brasil, 1990-1996.

\begin{tabular}{|c|c|c|c|c|c|c|c|c|c|}
\hline \multirow[t]{2}{*}{ Escolaridade } & \multicolumn{7}{|c|}{ Ano de diagnóstico } & \multirow[t]{2}{*}{ Taxa de variação anual (\%) } & \multirow[t]{2}{*}{$\mathrm{p}$} \\
\hline & 90 & 91 & 92 & 93 & 94 & 95 & 96 & & \\
\hline \multicolumn{10}{|l|}{ Região Norte } \\
\hline $10 \mathrm{Grau}$ & - & 46,7 & 74,1 & 78,0 & 81,0 & 77,8 & 80,2 & 8,61 & 0,103 \\
\hline $20 \mathrm{Grau}$ & - & 53,3 & 25,9 & 22,0 & 19,0 & 22,2 & 19,8 & $-14,69$ & 0,070 \\
\hline \multicolumn{10}{|c|}{ Região Nordeste } \\
\hline $20 \mathrm{Grau}$ & 28,9 & 26,2 & 28,7 & 27,5 & 22,4 & 24,5 & 22,3 & $-4,04$ & 0,025 \\
\hline \multicolumn{10}{|c|}{ Região Sudeste } \\
\hline 1o Grau & 82,2 & 83,5 & 81,7 & 82,7 & 82,3 & 82,0 & 83,0 & 0,002 & 0,991 \\
\hline $20 \mathrm{Grau}$ & 17,8 & 16,5 & 18,3 & 17,3 & 17,7 & 18,0 & 17,0 & 0,003 & 0,997 \\
\hline \multicolumn{10}{|l|}{ Região Sul } \\
\hline 1o Grau & 70,8 & 75,0 & 70,4 & 74,5 & 71,6 & 74,0 & 77,7 & 0,96 & 0,171 \\
\hline \multicolumn{10}{|c|}{ Região Centro-Oeste } \\
\hline 10 Grau & 70,5 & 83,0 & 72,2 & 78,1 & 81,9 & 82,9 & 82,4 & 2,15 & 0,106 \\
\hline 2 o Grau & 29,5 & 17,0 & 27,8 & 21,9 & 18,1 & 17,1 & 17,6 & $-6,82$ & 0,118 \\
\hline \multicolumn{10}{|l|}{ Brasil** } \\
\hline $10 \mathrm{Grau}$ & 79,8 & 81,4 & 79,2 & 80,6 & 80,4 & 80,3 & 81,6 & 0,19 & 0,373 \\
\hline $20 \mathrm{Grau}$ & 20,2 & 18,6 & 20,8 & 19,4 & 19,6 & 19,7 & 18,4 & $-0,79$ & 0,377 \\
\hline
\end{tabular}

* Os casos com escolaridade ignorada foram corrigidos por um modelo de análise discriminante.

** Incluídos casos da Região Norte de 1990. 
Iheres, desde o início da série temporal, as proporções no grupo de menor grau de escolaridade já ultrapassavam o valor de 70\% e apresentavam um comportamento, no período, de estabilidade.

Entre os casos do sexo masculino classificados em escolaridade " 1 o grau", as taxas de variação anual foram positivas e significativamente diferentes de zero, para todas as regiões, independente do método utilizado para alocação dos casos com escolaridade ignorada. A menor taxa de variação foi observada na Região Sudeste. As taxas de variação anual para as proporções de casos classificados em escoIaridade "2o grau", complementares às primeiras, foram negativas e significativamente diferentes de zero.

Para o sexo feminino, não foi observada qualquer diferença significativa nas taxas de variação anual para ambas as categorias de escolaridade estudadas em nenhuma das regiões, com exceção da Região Nordeste, que apresentou tendências de variação similares àquelas observadas para o sexo masculino, ao utilizarse o modelo discriminante.

As diferenças observadas a partir dos procedimentos que constam das Tabelas $1 a, 1 b, 2 a$ e $2 b$ foram pouco expressivas. O modelo discriminante alocou mais casos na categoria " 1 o grau" do que na " 2 o grau", para ambos os sexos e todas as regiões do país.

As taxas de incidência de AIDS para indivíduos com 20-69 anos de idade segundo categoria de escolaridade por região e ano de diagnóstico para os sexos masculino e feminino são apresentadas na Tabela 3 (a e b).

Para o Brasil, contata-se que, em 1989, para os homens, a razão entre as taxas de incidência de AIDS em cada categoria de escolaridade era maior do que 2, com risco maior entre os casos de "2o grau". A evolução de crescimento das taxas de incidência para aqueles com escolaridade de "1o grau", diferentemente do comportamento apresentado pelas taxas de incidência dos casos com escolaridade "2o grau", faz com que a razão entre as taxas de incidência por escolaridade se torne próxima de 1 em 1996. A evolução das taxas de incidência para aqueles com escolaridade de " 2 o grau" obedece à tendência inicial de aclive, seguida de declive gradual após 1992. As taxas de variação anual corroboram esses achados, já que foram significativamente diferentes de zero no grupo de "10 grau" e não significativas no grupo de " 20 grau".

Chamam atenção as elevadas taxas de variação anual observadas nas regiões Sul (30\%) e Centro-Oeste (28\%) entre os casos masculinos com grau de escolaridade " 10 grau". A Re- gião Sul foi a única que registrou também aumento relativo significativo entre os casos de "2o grau".

Para o sexo feminino, o comportamento das taxas de incidência de AIDS por escolaridade se diferencia daquele encontrado para o sexo masculino. Em primeiro lugar, as taxas de incidência, desde o início da série, são mais elevadas na categoria "o grau". Além disso, as taxas de incidência em ambas as categorias de escolaridade apresentam incremento anual de aproximadamente $25 \%$, ao longo do período estudado. As diferenças regionais são evidentes. De forma semelhante ao observado para os casos masculinos, a Região Sul é a que apresenta as maiores taxas de variação anual para os dois grupos de escolaridade. Nas Regiões Norte, Nordeste e Centro-Oeste, a taxa de variação anual é maior entre as mulheres com menor nível de instrução.

A visualização do comportamento temporal das taxas de incidência de AIDS por grau de escolaridade nas diferentes regiões é apresentada na Figura 1.

Entre os casos masculinos, a Região Sudeste apresenta um padrão peculiar, no qual as taxas de incidência de AIDS referentes à categoria "1o grau" ultrapassam, a partir de 1994, as taxas relativas à categoria "2o grau". As demais apresentam tendência no sentido de evoluir em alguns anos para padrão similar, já que se observa uma redução progressiva nas razões entre as taxas de incidência por escolaridade. Para o sexo feminino, as curvas que descrevem a evolução temporal das taxas de incidência por escolaridade mostram maior ritmo de crescimento entre as mulheres de menor escolaridade para todas as regiões, com exceção da Sudeste.

O padrão observado nas regiões Norte, Sul e Centro-Oeste mostra que as curvas para as mulheres com escolaridade mais baixa acabam por ultrapassar as das mulheres com maior instrução. Na Região Nordeste, até o último ano do período analisado, não se observa esse padrão, embora se perceba uma tendência nesse sentido. A Região Sudeste, apesar de, no período 1990-1996, ter apresentado curvas paralelas, com taxas superiores para os casos classificados no "1o grau", registrou comportamento similar de intercessão das curvas em anos anteriores (no início da epidemia nesta região). Esse comportamento está visualizado com maior detalhe na Figura 2. 
Taxas de incidência de AIDS (por 100.000) em homens de 20 a 69 anos de idade por grau de escolaridade e ano de diagnóstico e taxas de variação anual (\%) segundo as grandes regiões. Brasil, 1989-1996.

\begin{tabular}{|c|c|c|c|c|c|c|c|c|c|c|}
\hline \multirow[t]{2}{*}{ Escolaridade } & \multicolumn{8}{|c|}{ Ano de diagnóstico } & \multirow{2}{*}{$\begin{array}{l}\text { Taxa de variação } \\
\text { anual (\%) }\end{array}$} & \multirow[t]{2}{*}{$\mathrm{p}$} \\
\hline & 89 & 90 & 91 & 92 & 93 & 94 & 95 & 96 & & \\
\hline \multicolumn{11}{|c|}{ Região Norte** } \\
\hline $10 \mathrm{Grau}$ & - & - & 2,67 & 4,65 & 5,20 & 6,77 & 7,86 & 9,77 & 26,87 & 0,001 \\
\hline 2o Grau & - & - & 14,94 & 17,77 & 17,88 & 22,62 & 20,81 & 18,26 & 5,01 & 0,173 \\
\hline \multicolumn{11}{|c|}{ Região Nordeste } \\
\hline 1o Grau & 2,32 & 3,12 & 4,56 & 6,05 & 7,57 & 8,47 & 8,85 & 10,56 & 20,92 & 0,001 \\
\hline $20 \mathrm{Grau}$ & 19,42 & 21,00 & 26,47 & 28,50 & 23,76 & 24,83 & 23,16 & 24,80 & 0,33 & 0,875 \\
\hline \multicolumn{11}{|c|}{ Região Sudeste } \\
\hline 1ㅇ G rau & 19,88 & 28,40 & 36,65 & 46,08 & 49,86 & 52,19 & 52,79 & 54,62 & 10,63 & 0,041 \\
\hline $20 \mathrm{Grau}$ & 32,71 & 39,61 & 44,53 & 52,06 & 52,69 & 50,33 & 48,22 & 43,35 & 1,42 & 0,531 \\
\hline \multicolumn{11}{|l|}{ Região Sul } \\
\hline $10 \mathrm{Grau}$ & 3,10 & 7,13 & 11,58 & 14,67 & 20,24 & 22,90 & 26,82 & 30,19 & 25,99 & 0,000 \\
\hline $20 \mathrm{Grau}$ & 16,21 & 22,48 & 30,86 & 30,30 & 34,67 & 39,50 & 38,48 & 39,62 & 8,97 & 0,005 \\
\hline \multicolumn{11}{|c|}{ Região Centro-Oeste } \\
\hline 10 Grau & 3,82 & 5,61 & 12,09 & 17,07 & 19,38 & 24,27 & 29,29 & 25,85 & 27,00 & 0,005 \\
\hline $20 \mathrm{Grau}$ & 15,86 & 22,41 & 32,49 & 37,41 & 27,20 & 23,72 & 33,11 & 29,02 & 1,28 & 0,751 \\
\hline \multicolumn{11}{|l|}{ Brasil } \\
\hline 10 Grau & 10,09 & 14,70 & 19,86 & 25,24 & 28,32 & 30,37 & 31,73 & 33,37 & 17,58 & 0,001 \\
\hline $20 \mathrm{Grau}$ & 25,52 & 31,30 & 36,91 & 41,69 & 41,22 & 40,80 & 39,62 & 36,97 & 4,95 & 0,056 \\
\hline
\end{tabular}

* Os casos com escolaridade ignorada foram corrigidos por um modelo de análise discriminante.

** Os casos da Região N orte somente foram analisados para o período 1991-1996.

\section{Tabela 3b}

Taxas de incidência de AIDS (por 100.000) em mulheres de 20 a 69 anos de idade por grau de escolaridade e ano de diagnóstico e taxas de variação anual (\%) segundo as grandes regiões. Brasil, 1990-1996.

\begin{tabular}{|c|c|c|c|c|c|c|c|c|c|}
\hline \multirow[t]{2}{*}{ Escolaridade } & \multicolumn{7}{|c|}{ Ano de diagnóstico } & \multirow[t]{2}{*}{ Taxa de variação anual (\%) } & \multirow[t]{2}{*}{$\mathrm{p}$} \\
\hline & 90 & 91 & 92 & 93 & 94 & 95 & 96 & & \\
\hline \multicolumn{10}{|c|}{ Região Norte** } \\
\hline $10 \mathrm{Grau}$ & - & 0,41 & 1,12 & 1,73 & 2,67 & 2,82 & 3,74 & 50,38 & 0,005 \\
\hline $20 \mathrm{Grau}$ & - & 1,79 & 1,51 & 1,87 & 2,40 & 3,09 & 3,54 & 18,06 & 0,007 \\
\hline \multicolumn{10}{|c|}{ Região Nordeste } \\
\hline $20 \mathrm{Grau}$ & 1,09 & 1,60 & 2,41 & 2,92 & 2,54 & 3,37 & 4,29 & 22,38 & 0,001 \\
\hline \multicolumn{10}{|c|}{ Região Sudeste } \\
\hline 1o Grau & 5,77 & 8,29 & 12,26 & 15,13 & 16,17 & 18,31 & 21,12 & 22,88 & 0,001 \\
\hline $20 \mathrm{Grau}$ & 2,98 & 3,91 & 6,55 & 7,55 & 8,28 & 9,57 & 10,32 & 22,88 & 0,001 \\
\hline \multicolumn{10}{|l|}{ Região Sul } \\
\hline 10 Grau & 1,48 & 3,21 & 4,30 & 6,45 & 7,50 & 9,90 & 13,26 & 39,79 & 0,000 \\
\hline \multicolumn{10}{|c|}{ Região Centro-Oeste } \\
\hline 10 Grau & 1,82 & 4,42 & 4,28 & 6,04 & 9,49 & 8,93 & 12,14 & 32,58 & 0,001 \\
\hline 2 으 Grau & 1,83 & 2,20 & 4,01 & 4,17 & 5,21 & 4,58 & 6,52 & 21,90 & 0,002 \\
\hline \multicolumn{10}{|l|}{ Brasil } \\
\hline $10 \mathrm{Grau}$ & 3,09 & 4,75 & 6,80 & 8,64 & 9,60 & 10,99 & 13,31 & 25,73 & 0,000 \\
\hline $20 \mathrm{Grau}$ & 2,25 & 3,13 & 5,14 & 5,97 & 6,72 & 7,77 & 8,65 & 24,48 & 0,001 \\
\hline
\end{tabular}

* Os casos com escolaridade ignorada foram corrigidos por um modelo de análise discriminante.

** Os casos da Região Norte somente foram analisados para o período 1991-1996. 
Figura 1

Taxas de incidência de AIDS (por 100.000) para indivíduos de 20 a 69 anos de idade segundo grande região*, sexo** e grau de escolaridade*** por ano de diagnóstico. Brasil, 1989-96.
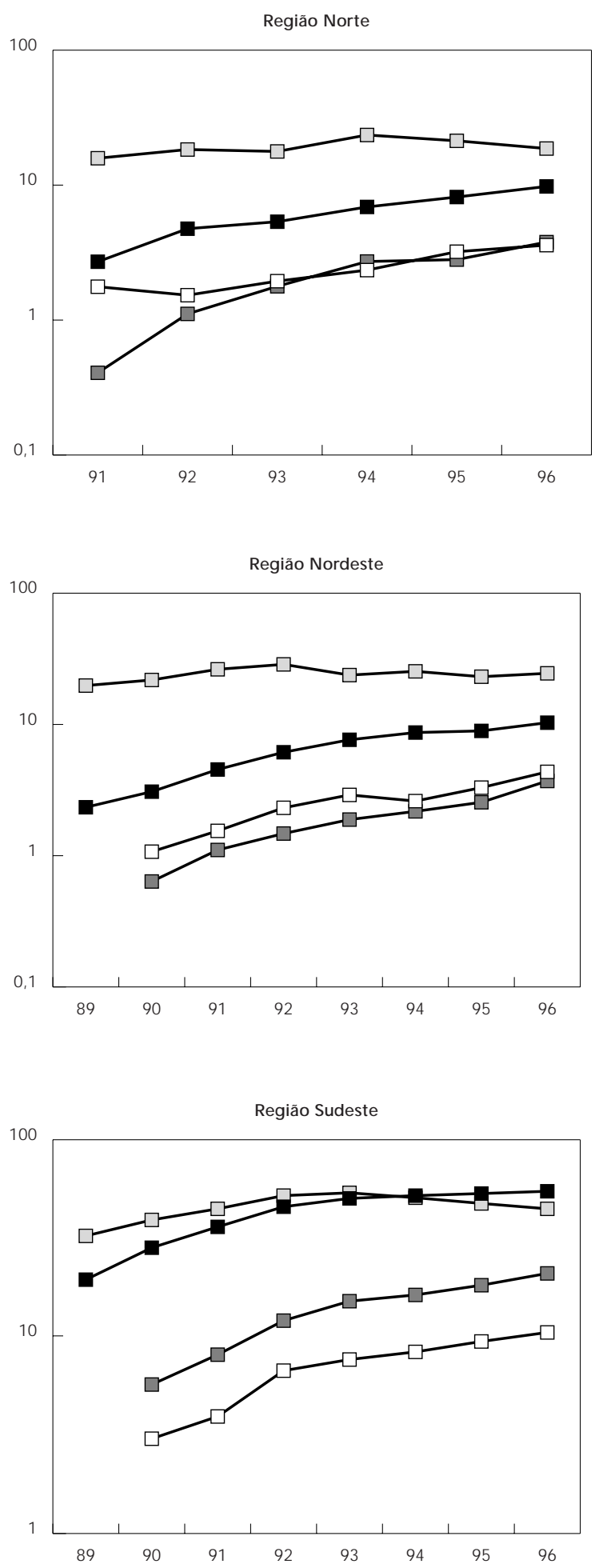

(continua)

\section{Discussão}

A presente análise mostra, de forma consistente, que a epidemia de AIDS no Brasil se iniciou nos estratos sociais de maior escolaridade, com progressiva disseminação para os estratos sociais de menor escolaridade. Esse fato foi evidenciado tanto pela variação na proporção de casos como pelas taxas de incidência, em todas as regiões do país, para ambos os sexos.

As propostas de análise da epidemia de AIDS no Brasil que tentam incorporar a escolaridade até hoje desenvolvidas se viram às voltas com as dificuldades decorrentes da grande proporção de casos com escolaridade ignorada (ver os diversos artigos coligidos em CNDST/ AIDS, 1997). Nesse sentido, procedeu-se no presente trabalho a uma análise discriminante dos casos com escolaridade informada que permitiu reclassificar os casos com escolaridade ignorada e calcular taxas de incidência por grau de instrução.

Pôde-se perceber que, diferentemente de al gumas suposi ções correntes, o risco a que estão expostos os indivíduos do sexo masculino de maior escolaridade ainda é superior àquele observado nos indivíduos de menor escolaridade, com a exceção da Região Sudeste, onde tem lugar a epidemia mais antiga do país (Ministério da Saúde, 1999), e na Região CentroOeste, onde os dois grupos apresentam riscos comparáveis. Já para o sexo feminino, o quadro é claramente distinto, com maior risco entre as mulheres de menor escolaridade, nas regiões Sudeste e Centro-Oeste, risco, grosso modo, similar entre as duas categorias de escolaridade nas regiões Norte e Sul, e risco ampliado entre as mulheres com maior escolaridade na Região Nordeste.

Levando em conta análise anterior que incorpora a escolaridade ao estudo das categorias de exposição (Fonseca \& Castilho, 1997), é possível inferir que o declínio na participação proporcional da categoria de transmissão "homossexual" (ver artigo de Szwarcwald et al., neste fascículo) - onde foram observadas mai ores proporções de casos entre pessoas com maior escolaridade - está associado ao declínio da participação proporcional dos casos entre pessoas com maior escolaridade, especialmente no Sudeste.

Esta hipótese é reforçada pela constatação de que nos casos femininos, onde a transmissão heterossexual é predominante, observamse taxas elevadas de inci dência de AIDS para a categoria de mais baixa instrução.

Outro fator que parece ter influído no incremento dos casos registrados entre aqueles 
de menor escolaridade nas regiões Sudeste e Sul, especialmente entre 1989 e 1992, foi o aumento da participação proporcional da categoria de exposição "usuário de drogas injetáveis", que, segundo a supracitada análise de Fonseca \& Castilho (1997), pertencem, majoritariamente, a estratos de escolaridade mais baixa.

O trabalho corrobora achados de estudos anteriores que sublinham a heterogeneidade da epidemia no Brasil (Bastos \& Barcellos, 1995; Barcellos \& Bastos, 1996; Szwarcwald et al., 1997; Szwarcwald et al., 1998). Os resultados dessa análise indicam que a epidemia de AIDS na Região Sudeste, por ser mais antiga e extensa, apresenta-se em fase mais avançada, enquanto as regiões Sul e Centro-Oeste, em uma situação intermediária. Nas regiões Norte e Nordeste, as taxas de incidências mais baixas em magnitude e os valores mais altos nas populações de maior escolaridade expressam uma fase inicial da epidemia.

Ainda que reconhecendo a necessidade de utilização conjunta de indicadores diversos para uma mensuração mais precisa do status sócio-econômico dos casos de AIDS em análise, cabe ponderar que a escolaridade está menos sujeita às influências da evolução da própria infecção sobre o acesso ao emprego ou proporção dos gastos com saúde no conjunto da renda individual e familiar (Kass et al., 1994).

Além disso, há uma relevante superposição cronológica entre a epidemia de AIDS e as transformações do capitalismo em geral e do modelo econômico brasileiro em particular (ver artigo de Parker \& Camargo Jr. neste fascículo), com um processo, em curso, de profunda reestruturação do mercado de trabal ho e aumento do desemprego, com repercussões óbvias sobre a estrutura ocupacional do país e da fração da renda nacional percebida por cada uma das diferentes categorias ocupacionais. Portanto, ainda que seja possível tratar, de forma similar a que fizemos para a escolaridade, os dados constantes das fichas de notificação referentes à ocupação, há que se ter cautela ao lidar com um indicador muito menos estável, tanto do ponto de vista individual como macro-econômico.

Entre as limitações do presente estudo, cita-se basicamente o uso de dados secundários, que, em análises epidemiológicas, pode dar origem a erros pela variação dos critérios de definição de caso ao longo da série temporal, pela má qualidade do preenchimento das informações, além do subenumeração dos casos de fato ocorridos (Brookmeyer \& Gail, 1994). Os dados aqui utilizados foram fornecidos pela Coordenação Nacional de DST e AIDS do Ministério
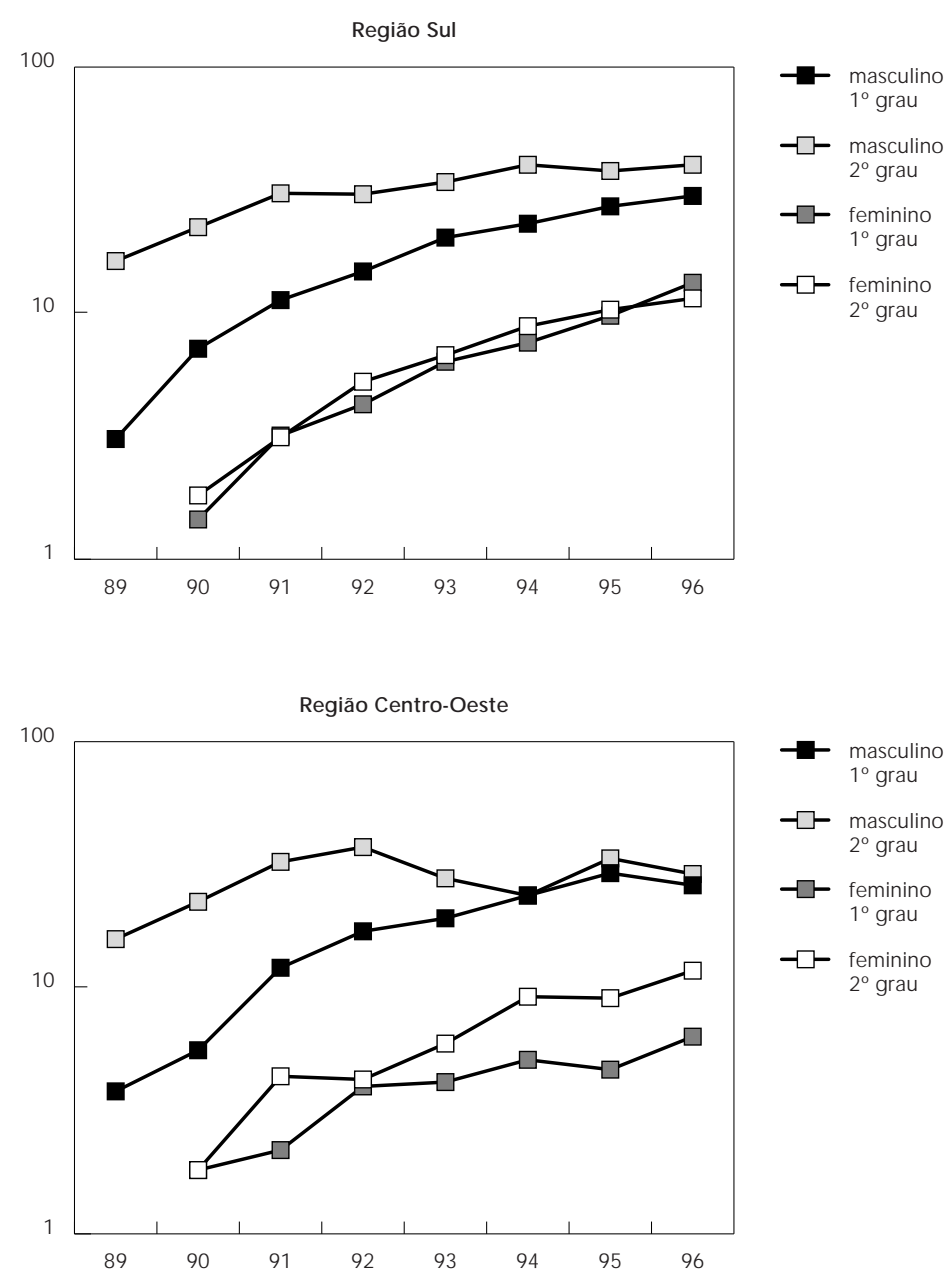

* Para a Região Norte foi analisada a tendência para o período de 1991-96. ** Para o sexo feminino foi analisada a tendência para o período de 1990-96. *** Os casos com escolaridade ignorada foram corrigidos por um modelo de análise discriminante.

da Saúde, responsável tanto pela sistematização das notificações como pela padronização da definição de caso de AIDS, e abrangeram somente até o ano de 1996 para diminuir o efeito do atraso de notificação.

Quanto à qualidade do preenchimento das informações relativas aos casos em estudo, particularmente no caso desta investigação, cabe observar que a elevada proporção de "informação ignorada" no campo "escolaridade" pode acarretar vícios nas análises das tendências temporais, comprometendo os resultados. Procurou-se contornar essa limitação, utilizandose um modelo discriminante para correção das informações ignoradas. 
Figura 2

Taxas de incidência de AIDS (por 100000) para indivíduos de 20 a 69 anos de idade segundo sexo

e grau de escolaridade* por ano de diagnóstico. Região Sudeste, 1989-96.

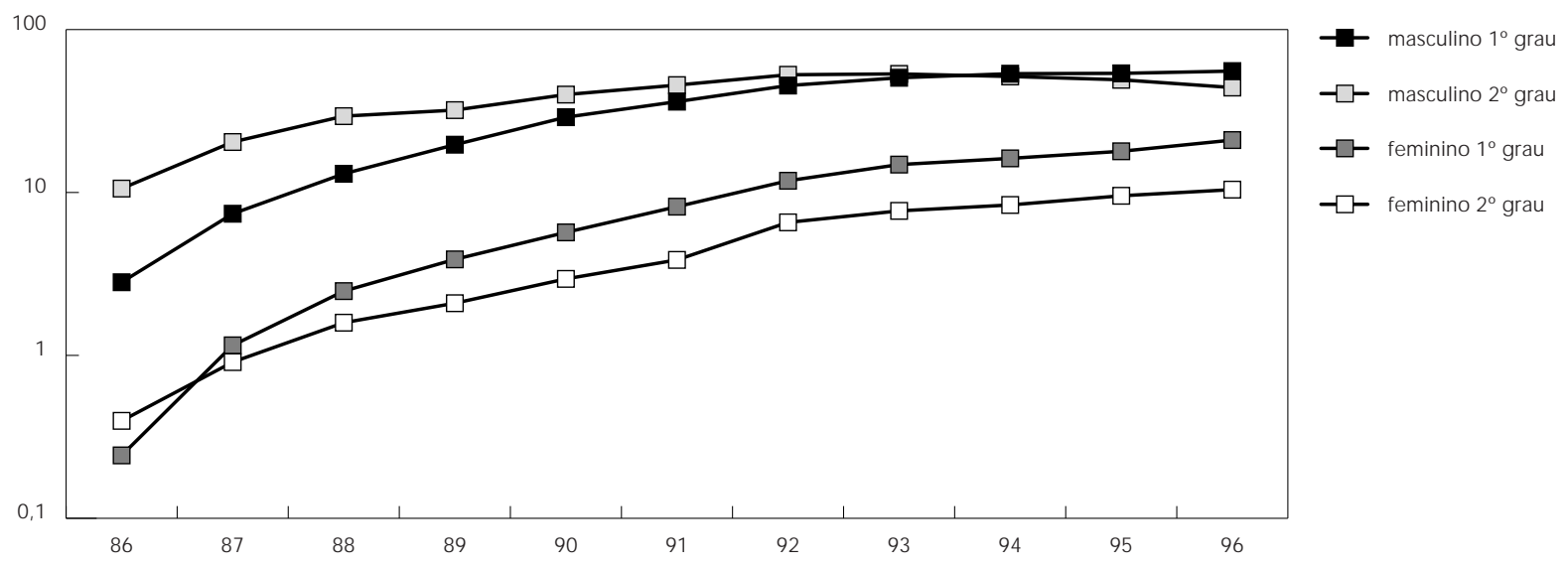

* Os casos com escolaridade ignorada foram corrigidos por um modelo de análise discriminante.

Outra limitação com que se deparam os que analisam os dados referentes à escolaridade refere-se às dificuldades referentes às categorizações dos graus de escolaridade utilizadas no banco original, como a não especificação da série efetivamente completada do ensino fundamental (1o grau) e a flutuação ao longo da série sob análise dos critérios de classificação, o que fez com que tivéssemos de reunir em uma única categoria todos os indivíduos com escolaridade de até 8 anos de estudo, certamente contribuindo para a baixa sensibilidade desse indicador como mensurador de pobreza, principalmente em um país com grande concentração de pessoas que não têm o 1o grau completo, por causa, entre outros fatores, de níveis muito elevados de evasão escolar nas primeiras séries do ensino fundamental (Saboia, 1998).
Apesar das limitações impostas pela utilização de dados secundários, é notório que a epidemia de AIDS vem atingindo paulatinamente populações em desvantagem sócio-econômica. Em um país marcado pelas diferenças sociais, o risco crescente de pessoas pertencentes aos segmentos populacionais menos favorecidos ante o HIV/ AIDS certamente lança novos desafios à sociedade e aos profissionais engajados no controle da doença. As medidas de prevenção devem contemplar, de forma culturalmente sensível, grupos populacionais de todas as regiões do país, com diferentes graus de acesso aos serviços e engajamento nas instâncias da sociedade civil organizada, e, certamente, com distintos níveis de compreensão e percepção de sua vulnerabilidade diante do HIV/ AIDS, e diferentes possibilidades de participar e influir nessas e nas demais políticas públicas e movimentos sociais.

\section{Referências}

ARBER, S., 1996. Integrating nonemployment into research on health inequalities. International Journal of Health Services, 26:445-481.

BARBOSA, M. T. S. \& STRUCHINER, C. J., 1997. Estimativas do número de casos de AIDS: Comparação de métodos que corrigem o atraso da notificação. In: A Epidemia de AIDS no Brasil: Situação eTendências (Coordenação Nacional de DST e AIDS, org.), pp. 15-26, Brasília: Ministério da Saúde. 
BARCELLOS, C. \& BASTOS, F. I., 1996. Redes sociais e difusão da AIDS no Brasil. Boletín de la Oficina Sanitaria Panamericana, 121:11-24.

BASTOS, F. I. \& BARCELLOS, C., 1995. A geografia social da AIDS no Brasil. Revista de Saúde Pública, 29:52-62.

BROOKMEYER, R. \& GAIL, M. H., 1994. AIDS Epidemi ology: A Quantitative Approach. New York: Oxford University Press.

CAIRNEY, J. \& ARNOLD, R., 1998. Socioeconomic position, lifestyle and health among Canadians aged 18 to 64: A multi-condition approach. Canadian Journal of Public Health, 89:208-212.

CNDST/ AIDS (Coordenação Nacional de DST e AIDS), 1997. A Epidemia de AIDS no Brasil: Situação e Tendências. Brasília: Ministério da Saúde.

EAMES, M.; BEN-SHLOMO, Y. \& MARMOT, M. G., 1993. Social deprivation and premature mortality: Regional comparison across England. BMJ, 307: 1097-1102.

FONSECA, M. G. P. \& CASTILHO, E. A., 1997. Os casos de AIDS entre os usuários de drogas injetáveis. Brasil, 1980-1997. Boletim Epidemiológico - AIDS, X(2):6-14, Semana Epidemiológica 23 a 35, junho/agosto.

FONSECA, M. G. P.; SILVA, N. V. \& HASENBALG, C., 1999. Saúde e Desigualdades Social e Regional no Brasil, 1996/1997. Rio de Janeiro: Instituto Universitário de Pesquisas do Rio de Janeiro. (mimeo.)

GULLIFORD, M. C. \& MAHABIR, D., 1998. Social inequalities in morbidity from diabetes mellitus in public primary care clinics in Trinidad and Tobago. Social Scienceand Medicine, 46:137-144.

HOSMER, D. W. \& LEMESHOW, S., 1989. Applied Logistic Regression. New York: John Wiley \& Sons.

KASS, N. E.; MUÑOZ, A.; CHEN, B.; ZUCCONI, S. L.; BING, E. G.; HENNESSY, M. \& THE MULTICENTER AIDS COHORT STUDY, 1994. Changes in employment, insurance, and income in relation to HIV status and disease progression. The Multicenter AIDS Cohort Study. Journal of Acquired Immune Deficiency Syndromes, 7:86-91

KUNST, A. E. \& MACKENBACH, J. P., 1994a. The size of mortality differences associated with educational level in nine industrialized countries. American Journal of Public Health, 84:932-937.

KUNST, A. E. \& MACKENBACH, J. P., 1994b. Equity in Health Target. Measuring Socio-Economic Inequalities in Health. WHO Regional Office for Europe. Kopenhagen: World Health Organization.

LUEPKER, R. V.; ROSAM OND, W. D.; MURPHY, R.; SPRAFKA, J. M.; FOLSOM, A. R.; MCGOVERN, P. G. \& BLACKBURN, H., 1993. Socioeconomic status and coronary heart disease risk factor trends. The Minnesota Heart Survey. Circulation, 88:21722179.

MACHARELLI, C. A. \& OLIVEIRA, L. R., 1991. Perfil do risco de óbito de crianças menores de um ano residentes em localidade do Estado de São Paulo, Brasil, 1987. Revista de Saúde Pública, 25:121-128.

MACKENBACH, J. P.; KUNST, A. E.; CAVELAARS, A. E. J. M.; GROENHOF, F.; GEURTS, J. J. M. \& THE EU WORKING GROUP ON SOCIOECONOMIC INEQUALITIES IN HEALTH, 1997. Socioeconomic inequalities in morbidity and mortality in western Europe. Lancet, 349:1655-1659.
MANN, J. M.; TARANTOLA, D. \& NETTER, T. W., 1992. AIDS in theWorld. Cambridge: Harvard University Press.

MS (Ministério da Saúde), 1999. Boletim Epidemiológico - AIDS, XII (2), Semana Epidemiológica 09 a 21 , março/maio.

MUSTARD, C. A.; DERKSEN, S.; BERTHELOT, J. M .; WOLFSON, M. \& ROOS, L. L., 1997. Age-specific education and income gradients in morbidity and mortality in a Canadian province. Social Science and Medicine, 45:383-397.

PAPPAS, G.; QUEEN, S.; HADDEN, W. \& FISHER, G., 1993. The increasing disparity in mortality between socioeconomic groups in the United States, 1960 and 1986. New England Journal of Medicine, 329:103-109.

SABOIA, A. L., 1998. Situação educacional dos jovens. In: Jovens Acontecendo nas Tril has das Políticas Públicas (Comissão Nacional de População e Desenvolvimento - CNPD, org.), v. 2, pp. 135-148, Brasília: CNPD.

SLOGGETT, A. \& JOSHI, H., 1998. Deprivation indicators as predictors of life events 1981-1992 based on the UK NOS Iongitudinal study. Journal of Epidemiology and Community Health, 52:228-233.

SORLIE, P. D.; BACKLUND, E. \& KELLER, J. B., 1995. US mortality by economic, demographic, and social characteristics: The National Longitudinal Mortality Study. American Journal of Public Health, 85:949-956

SZWARCWALD, C. L.; BASTOS, F. I.; ANDRADE, C. L. T. \& CASTILHO, E. A., 1997. AIDS: O mapa ecológico do Brasil, 1982-1994. In: A Epidemia de AIDS no Brasil: Situação e Tendências (Coordenação Nacional de DST e AIDS, org.), pp. 27-44, Brasília: Ministério da Saúde.

SZWARCWALD, C. L.; BASTOS, F. I. \& CASTILHO, E. A., 1998. The dynamic of the AIDS epidemic in Brazil: A space-time analysis in the period 19871995. Brazilian Journal of Infectious Diseases, 2: 175-186.

UNAIDS (THE JOINT UNITED NATIONS PROGRAMME ON HIV/AIDS), 1999. UNAIDS 3rd Meeting of the Latin America and Caribbean Epidemiological Network, Abstracts, Cuernavaca: UNAIDS.

WALLACE, R., 1988. A synergism of plagues: "Planned shrinkage," contagious housing destruction and AIDS in the Bronx. Environmental Research, 47:133.

WALLACE, R., 1990. Urban desertification, public health and public order: "Planned shrinkage," violent death, substance abuse and AIDS in the Bronx. Social Scienceand Medicine, 31:801-813. 